\title{
A DYNAMIC WATERMARKING MODEL FOR EMBEDDING REDUCIBLE PERMUTATION GRAPHS INTO SOFTWARE
}

\author{
Ioannis Chionis, Maria Chroni, and Stavros D. Nikolopoulos \\ Department of Computer Science, University of Ioannina, GR-45110 Ioannina, Greece \\ \{ichionis, mchroni, stavros\}@cs.uoi.gr
}

Keywords: Software watermarking; watermark numbers; self-inverting permutations; reducible permutation graphs; encoding; decoding; graph embedding; call-graphs; control statement; opaque predicates, algorithms.

Abstract: $\quad$ Software watermarking involves embedding a unique identifier or, equivalently, a watermark value, within a software to discourage software theft; towards the embedding process, several graph theoretic watermarking algorithmic techniques encode the watermark values as graph structures and embed them in application programs. Recently, we presented an efficient codec system for encoding a watermark number $w$ as a reducible permutation graph $F\left[\pi^{*}\right]$ through the use of self-inverting permutations $\pi^{*}$. In this paper, we propose a dynamic watermarking model for embedding the watermark graph $F\left[\pi^{*}\right]$ into an application program $P$. The main idea behind the proposed watermarking model is a systematic use of appropriate calls of specific functions of the program $P$. More precisely, our model uses the dynamic call-graph $G\left(P, I_{k e y}\right)$ of the program $P$, taken by the specific input $I_{k e y}$, and the graph $F\left[\pi^{*}\right]$, and produces the watermarked program $P^{*}$ having the following key property: its dynamic call-graph $G\left(P^{*}, I_{\text {key }}\right)$ and the reducible permutation graph $F\left[\pi^{*}\right]$ are isomorphic graphs. Within this idea the program $P^{*}$ is produced by only altering appropriate real-calls of specific functions of the input program $P$. Moreover, the proposed watermarking model incorporates such properties which cause it resilient to attacks.

\section{INTRODUCTION}

The rapid growth of World Wide Web users, the ease of distributing fast and in the original form digital content through internet, as well as the lack of technical measures to assure the intellectual property right of owners, has led to the increasing of copyright infringement. Digital watermarking is a technique for protecting the intellectual property of any digital content. The idea of digital watermarking is the embedding of a unique identifier into the digital image, audio, or video data, software and text through the introduction of errors not detectable by human perception (Cox et al., 1996).

According to the recent Business Software Alliance (BSA) global software piracy study (BSA, 2011) over half of the worlds personal computer users - 57 percent - admit they pirate software. What is more, as the price of hardware drops and the price of licensed software goes up, piracy becomes more popular and lucrative. This fact has led to a more systematic work on protecting the intellectual property as can be seen from a recent research of World Intellec- tual Property Organization (WIPO) where there is a growth of intellectual property filings (WIPO, 2012).

Software Watermarking. Although digital watermarking has made considerable progress and become a popular technique for copyright protection of multimedia information (Cox et al., 1996), research on software watermarking has recently received sufficient attention. The patent by Davidson and Myhrvold (Davidson and Myhrvold, 1996) presented the first published software watermarking algorithm, where and other patents have been published lately (Rodriguez et al., 2010; Collberg et al., 2011; Horne et al., 2012). The major software watermarking algorithms currently available are based on several techniques, among which the register allocation (XiaoCheng and Zhiming, 2010), spread-spectrum (Zhang et al., 2011), opaque predicate (Arboit, 2002), abstract interpretation (Cousot and Cousot, 2004), dynamic path techniques (Collberg et al., 2004), code reorderings (Sharma et al., 2011) (Curran et al., 2003; Grover, 1997; Monden et al., 2000; Nagra and Thomborson, 2004; Qu and Potkonjak, 1998; Stern et al., 1999); see also, Collberg and Nagra (Collberg and 
Nagra, 2010) and (Zhang et al., 2003; Zhu et al., 2005; Sharma et al., 2011; Zhang et al., 2009) for an exposition of the main results.

The software watermarking problem can be described as the problem of embedding a structure $w$ into a program $P$ and, thus, producing a new program $P_{w}$, such that $w$ can be reliably located and extracted from $P_{w}$ even after $P_{w}$ has been subjected to code transformations such as translation, optimization and obfuscation (Myles and Collberg, 2006). More precisely, given a program $P$, a watermark $w$, and a key $k$, the software watermarking problem can be formally described by the following two functions: $\operatorname{embed}(P, w, k) \rightarrow P_{w}$ and $\operatorname{extract}\left(P_{w}, k\right) \rightarrow w$.

There are two main categories of watermarking algorithms namely static and dynamic algorithms (Collberg and Thomborson, 1999). A static watermark is stored inside program code in a certain format, and it does not change during the program execution. A dynamic watermark is built during program execution, perhaps only after a particular sequence of input. It might be retrieved by analyzing the data structures built when watermarked program is running. In other cases, tracing the program execution may be required. Further discussion of static and/or dynamic watermarking issues can be found in (Davidson and Myhrvold, 1996; Moskowitz and Cooperman, 1996; Venkatesan et al., 2001).

Having designed a static or dynamic software watermarking algorithm for embedding a watermark $w$ in to an application program $P$, producing thus the watermarked program $P_{w}$, it is very important to evaluate the watermarking algorithm under various criteria in order to gain information about its practical behavior. A software watermarking algorithm can be evaluated using several criteria (Collberg et al., 2009); we mention some of the most important:

- Data rate,

- Embedding overhead,

- Resistance to detection (stealth), and

- Resilience against transformations.

Graph-based Codecs and Attacks. Recently, several software watermarking algorithms have been appeared in the literature that encode watermarks as graph structures. In general, such encodings make use of an encoding function encode which converts a watermarking number $w$ into a graph $G$, encode $(w) \rightarrow G$, and also of a decoding function decode that converts the graph $G$ into the number $w$, $\operatorname{decode}(G) \rightarrow w$; we usually call the pair (encode, decode $)_{G}$ as graph codec system (Collberg et al., 2003). From a graph-theoretic point of view, we are looking for a class of graphs $\mathcal{G}$ and a corresponding codec (encode, $\mathrm{decode})_{\mathcal{G}}$ with the following properties which cause them resilience to attacks:

- Appropriate graph types: Graphs in $\mathcal{G}$ should be directed having such properties, i.e., nodes with small outdegree, so that matching real program graphs;

- High resiliency: The function $\operatorname{decode}(G)$ should be insensitive to small changes of $G$, i.e., insertions or deletions of a constant number of nodes or/and edges; that is, if $G \in \mathcal{G}$ and $\operatorname{decode}(G) \rightarrow$ $w$ then $\operatorname{decode}\left(G^{\prime}\right) \rightarrow w$ with $G^{\prime} \approx G$;

- Small size: The size $\left|P_{w}\right|-|P|$ of the embedded watermark should be small;

- Efficient codecs: The functions encode and decode should be computed in polynomial time.

Related Work. In 1996, Davidson and Myhrvold (Davidson and Myhrvold, 1996) proposed the first software watermarking algorithm which is static and embeds the watermark by reordering the basic blocks of a control flow-graph; note that a static watermark is stored inside program code in a certain format and it does not change during the program execution. Based on this idea, Venkatesan, Vazirani and Sinha (Venkatesan et al., 2001) proposed the first graphbased software watermarking algorithm which embeds the watermark by extending a method's control flow-graph through the insertion of a directed subgraph; it is also a static algorithm and is called VVS or GTW. In (Venkatesan et al., 2001) the construction of a directed graph $G$ (or, watermark graph $G$ ) is not discussed. Collberg et al. (Collberg et al., 2009) proposed an implementation of GTW, which they call $\mathrm{GTW}_{\mathrm{s} m}$, and it is the first publicly available implementation of the algorithm GTW. In $\mathrm{GTW}_{\mathrm{s} m}$ the watermark is encoded as a reducible permutation graph (RPG) (Collberg et al., 2003), which is a reducible control flow-graph with maximum out-degree of two, mimicking real code. Note that, for encoding integers the $\mathrm{GTW}_{\mathrm{s} m}$ method uses only those permutations that are self-inverting. The first dynamic watermarking algorithm (CT) was proposed by Collberg and Thomborson (Collberg and Thomborson, 1999); it embeds the watermark through a graph structure which is built on a heap at runtime.

Recently, the authors of this paper (authors' papers 2010 and 2011) extended the class of software watermarking algorithms and graph structures by proposing an efficient and easily implemented codec system for encoding watermark numbers as reducible permutation flow-graphs. They presented an algorithm which encodes a watermark number $w$ as 
self-inverting permutation $\pi^{*}$ and algorithms which encode the permutation $\pi^{*}$ as a reducible permutation flow-graph $F\left[\pi^{*}\right]$.

Our Contribution. Recently, we presented efficient and easily implemented algorithms for encoding numbers as reducible permutation flow-graphs (or, for short, RPG) through the use of self-inverting permutations (or, for short, $\mathrm{SiP}$ ). More precisely, we have designed an efficient method for encoding integers as self-inverting permutations (authors' papers 2010 and 2011), and algorithms for encoding a self-inverting permutation $\pi^{*}$ into a reducible permutation flowgraph $F\left[\pi^{*}\right]$; the graph $F\left[\pi^{*}\right]$ incorporates properties capable to match real program graphs, that is, it does not differ from the graph data structures built by real programs since its maximum outdegree does not exceed two and it has a unique root node so the program can reach other nodes from the root node.

In this paper, we propose a dynamic watermarking model for embedding the watermark graph $F\left[\pi^{*}\right]$ into an application program $P$. The main idea behind the proposed watermarking model is a systematic use of appropriate calls of specific functions of the program $P$. More precisely, our model uses the dynamic call-graph $G\left(P, I_{k e y}\right)$ of the program $P$, taken by the specific input $I_{k e y}$, and the graph $F\left[\pi^{*}\right]$, and produces the watermarked program $P^{*}$ having the following key property: its dynamic call-graph $G\left(P^{*}, I_{k e y}\right)$ and the reducible permutation graph $F\left[\pi^{*}\right]$ are isomorphic graphs. Within this idea the program $P^{*}$ is produced by only altering appropriate real-calls of specific functions of the input program $P$. Moreover, the proposed watermarking model incorporates such properties which cause it resilient to attacks.

Road Map. The paper is organized as follows: In Section 2 we establish the notation and related terminology, and present background results. In Section 3 we present our dynamic watermarking model; we first describe its data and operational components and then the embedding algorithm Encode_SIP.to.RPG and the extracting algorithm Encode_SIP.to.RPG. Finally, in Section 4 we conclude the paper and discuss properties of our watermarking model and possible future extensions.

\section{BACKGROUND RESULTS}

In this section, we present basic components and background results that are used in the design of our watermarking model. In particular, we present the main components namely self-inverting permutations (or, for short, $\mathrm{SiP}$ ) and reducible permutation graphs (or, for short, RPG) of our previous work on encoding numbers as graph structures. In fact, we concentrate on the properties of the RPG $F\left[\pi^{*}\right]$ which encodes a $\operatorname{SiP} \pi^{*}$ and also on the encoding and extracting algorithms. We also briefly discuss properties of dynamic call-graphs which are used as key-objects in our watermarking model for embedding the graph $F\left[\pi^{*}\right]$ into an application program.

\subsection{Encode Numbers as RPGs}

We consider finite graphs with no multiple edges. For a graph $G$, we denote by $V(G)$ and $E(G)$ the vertex set and edge set of $G$, respectively. We also consider permutations over the set $N_{n}=\{1,2, \ldots, n\}$.

\section{A. Self-inverting Permutation (SiP)}

Let $\pi$ be a permutation over the set $N_{n}$. We think of permutation $\pi$ as a sequence $\left(\pi_{1}, \pi_{2}, \ldots, \pi_{n}\right)$, so, for example, the permutation $\pi=(1,4,2,7,5,3,6)$ has $\pi_{1}=1, \pi_{2}=4$, etc. Notice that $\pi_{i}^{-1}$ is the position in the sequence of the number $i$; in our example, $\pi_{4}^{-1}=2, \pi_{7}^{-1}=4, \pi_{3}^{-1}=6$, etc.

Definition 2.1. Let $\pi=\left(\pi_{1}, \pi_{2}, \ldots, \pi_{n}\right)$ be a permutation over the set $N_{n}$. The inverse of $\pi$ is the permutation $\tau=\left(\tau_{1}, \tau_{2}, \ldots, \tau_{n}\right)$ with $\tau_{\pi_{i}}=\pi_{\tau_{i}}=i$. A selfinverting permutation (or, involution) is a permutation that is its own inverse: $\pi_{\pi_{i}}=i$.

Notation 2.1. Throughout the paper we denote a selfinverting permutation $\pi$ over the set $N_{n}$ as $\pi^{*}$;

By definition, every permutation has a unique inverse, and the inverse of the inverse is the original permutation. Clearly, a permutation is a self-inverting permutation iff all its cycles are of length 1 or 2; hereafter, we shall denote a 2 -cycle by $c=(x, y)$ and by 1 -cycle as $c=(x)$, or, equivalently, $c=(x, x)$.

\section{B. Reducible Permutation Graphs (RPG)}

A flow-graph is a directed graph $F$ with an initial node $s$ from which all other nodes are reachable. A directed graph $G$ is strongly connected when there is a path $x \rightarrow y$ for all nodes $x, y$ in $V(G)$. A node $u$ is an entry for a subgraph $H$ of the graph $G$ when there is a path $p=\left(y_{1}, y_{2}, \ldots, y_{k}, x\right)$ such that $p \cap H=\{x\}$.

Definition 2.2. A flow-graph is reducible when it does not have a strongly connected subgraph with two (or more) entries.

There are at least three other equivalent defini- 
tions, as Theorem 2.1 shows. Those definitions use a few more graph-theoretic concepts. An edge $(x, x)$ (for some node $x$ ) is a cycle-edge. A depth first search (DFS) of a flow-graph partitions its edges into tree edges (making up a spanning tree known as a DFS tree), forward edges (pointing to a successor in the spanning tree), back edges (pointing to a predecessor in the spanning tree, plus cycle-edges), and cross edges (the others). Tree edges, forward edges, and cross edges form a dag known as a DFS dag.

Theorem 2.1. (Hecht and Ullman, 1972; Hecht and Ullman, 1974): Let $F$ be a flow-graph. The following three statements are equivalent:

(1) the graph $F$ is reducible;

(2) the graph $F$ has a unique DFS dag;

(3) the graph $F$ can be transformed into a single node by repeated application of the transformations $T_{1}$ and $T_{2}$, where $T_{1}$ removes a cycle-edge, and $T_{2}$ picks a non-initial node $y$ that has only one incoming edge and glue nodes $x$ and $y$.

The reducible permutation graph $F\left[\pi^{*}\right]$ is directed with descending ordering on its nodes $V(G)=\{s=$ $\left.u_{n+1}, u_{n}, \ldots, u_{1}, u_{0}=t\right\}$. Throughout the paper, we shall call the edge $\left(u_{i}, u_{j}\right)$ forward if $i>j$ while we shall call $\left(u_{i}, u_{j}\right)$ backward if $i<j$.

\section{Codec Algorithms}

In (authors' paper, 2010) we introduced the notion of bitonic permutations and we presented two algorithms, namely Encode_W.to.SiP and Decode_SiP.to.W, for encoding an integer $w$ into an self-inverting permutation $\pi^{*}$ and extracting it from $\pi^{*}$; see also (authors paper, 2011). We have actually proved the following results.

Theorem 2.2. Let $w$ be an integer and let $b_{1} b_{2} \cdots b_{n}$ be the binary representation of $w$. The algorithm Encode_W.to.SiP encodes the number $w$ in a selfinverting permutation $\pi^{*}$ of length $2 n+1$ in $O(n)$ time and space.

Theorem 2.3. Let $\pi^{*}$ be a self-inverting permutation of length $n$ which encodes an integer $w$ using the algorithm Encode_W.to.SiP. The algorithm Decode_SiP.to. W correctly decodes the permutation $\pi^{*}$ in $O(n)$ time and space.

Recently, we have presented an efficient and easily implemented algorithm for encoding numbers as reducible permutation flow-graphs through the use of self-inverting permutations (authors' paper, 2012).
The watermark number $w=4$

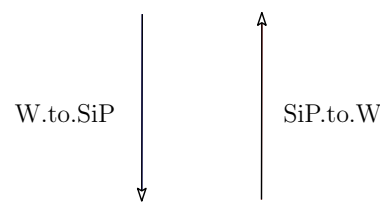

$$
\pi^{*}=(4,7,6,1,5,3,2)
$$
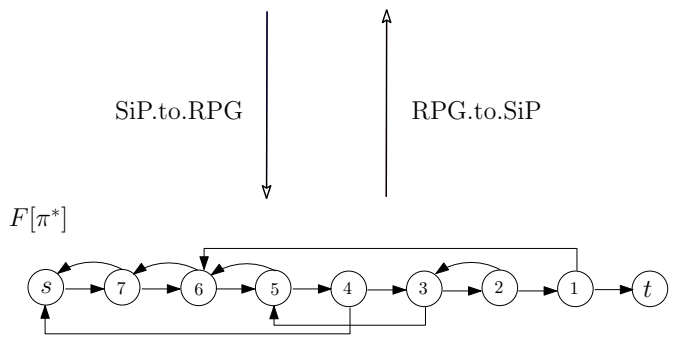

Figure 1: The main data components used by our codec algorithms (i.e., watermark $w, \operatorname{SiP} \pi^{*}$, and RPG $F\left[\pi^{*}\right]$ ) and a flow of the process of encoding a watermark number $w$ into the graph $F\left[\pi^{*}\right]$ and extracting it from $F\left[\pi^{*}\right]$.

In particular, we have proposed the algorithm Encode_SiP.to.RPG, which encodes the selfinverting permutation $\pi^{*}$ as a reducible permutation flow-graph $F\left[\pi^{*}\right]$ by exploiting domination relations on the elements of $\pi^{*}$ and using an efficient DAG representation of $\pi^{*}$. We also proposed the decoding algorithm Decode_RPG.to.SIP, which extract the self-inverting permutation $\pi^{*}$ from $F\left[\pi^{*}\right]$ by converting first the graph $F\left[\pi^{*}\right]$ into a directed tree $T\left[\pi^{*}\right]$ and then applying DFS-search on $T\left[\pi^{*}\right]$.

The whole encoding process takes $O(n)$ time and requires $O(n)$ space, where $n$ is the length of the permutation $\pi^{*}$. The decoding process takes time and space linear in the size of the flow-graph $F\left[\pi^{*}\right]$, that is, the algorithm Decode_RPG.to.SIP takes $O(n)$ time and space. Our results presented in (authors' paper, 2012) are summarized in the following theorems.

Theorem 2.4. Let $\pi^{*}$ be a self-inverting permutation over the set $N_{n}$. The algorithm Encode_SiP.to.RPG encodes the permutation $\pi^{*}$ into a reducible permutation graph $F\left[\pi^{*}\right]$ in $O(n)$ time and space.

Theorem 2.5. Let $F\left[\pi^{*}\right]$ be a reducible permutation graph of order $O(n)$ produced by the algorithm Encode_SiP.to.RPG. The algorithm Encode_RPG.to.SiP correctly extracts the permutation $\pi^{*}$ from $F\left[\pi^{*}\right]$ in $O(n)$ time and space.

Figure 1 depicts the main data components used by our codec algorithms, i.e., the watermark number 
$w$, the $\operatorname{SiP} \pi^{*}$, and the RPG $F\left[\pi^{*}\right]$. The same figure shows a flow of the process of encoding a watermark number $w$ into the graph $F\left[\pi^{*}\right]$ and extracting it from $F\left[\pi^{*}\right]$ through the use of self-inverting permutations.

\subsection{Dynamic Call-graphs}

A call-graph is a directed graph that represents calling relationships between program units in a computer program. Specifically, the nodes of a call-graph represent functions, procedures, classes, or similar program units and each edge $\left(f_{i}, f_{j}\right)$ indicates that function $f_{i}$ calls function $f_{j}$; function $f_{i}$ is called caller and function $f_{j}$ is called callee.

Call-graphs can be divided in two main classes of graphs, namely static and dynamic.

A static call-graph is the structure describing those invocations that could be made from one program unit to another in any possible execution of the program (Xie and Notkin, 2002). The static call-graph can be determined from the program source code; we mention that, its construction is a time consuming process specifically in the case of large scale softwares (Graham et al., 1982).

A dynamic call-graph $G$ is a directed graph that includes invocations of caller-callee pairs, over an execution of the program $P$. A dynamic call-graph can be considered as one instance of the corresponding static call-graph for a specific input sequence $I$. The call-graph $G$ is the key data structure that dynamic optimizers use to analyze and optimize whole-program behavior. Such a graph can be extracted by a profiler. It is fair to mention that the construction of a dynamic call-graph $G$ of a program $P$ is not a time consuming process even if $P$ is a large scale software.

Throughout the paper we denote a call-graph $G$ of the program $P$ over the input $I$ as $G(P, I)$. Figures 2(a) depicts the structure of the dynamic callgraph $G\left(P, I_{k e y}\right)$ of an application program $P$ with input $I_{k e y}$.

\section{THE DYNAMIC WATERMARKING MODEL}

Having encoded a watermark number $w$ as reducible permutation graph $F\left[\pi^{*}\right]$, let us now propose a dynamic watermarking model based on which we can efficiently watermark an application program $P$ by embedding the graph $F\left[\pi^{*}\right]$ into $P$ producing thus the watermarked program $P^{*}$.

The main idea behind the proposed dynamic watermarking model is the use of the dynamic call-

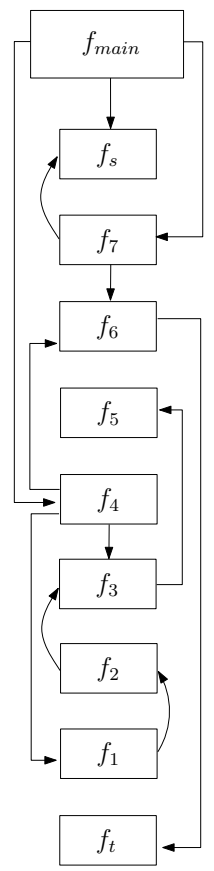

(a)

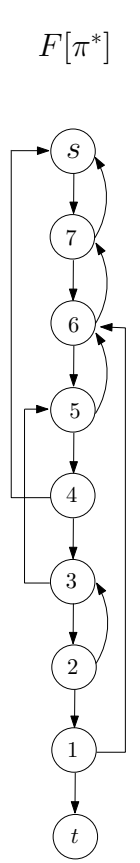

(b)

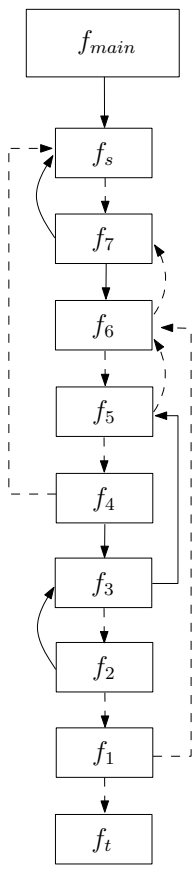

(c)
Figure 2: (a) The dynamic call-graph $G\left(P, I_{k e y}\right)$ of an application program $P$. (b) The reducible permutation graph $F\left[\pi^{*}\right]$. (c) The dynamic call-graph $G\left(P^{*}, I_{k e y}\right)$ of the watermarked program $P^{*}$.

graph $G\left(P, I_{k e y}\right)$ of the program $P$, taken by the specific input $I_{k e y}$, and the graph $F\left[\pi^{*}\right]$ in order to produce the watermarked program $P^{*}$ having the following key property: its dynamic call-graph $G\left(P^{*}, I_{\text {key }}\right)$ and the reducible permutation graph $F\left[\pi^{*}\right]$ are isomorphic graphs. Within this idea the program $P^{*}$ is produced by only altering appropriate real-calls of specific functions of the input program $P$.

Figure 2 shows the dynamic call-graph $G\left(P, I_{k e y}\right)$ of an application program $P$, the reducible permutation graph $F\left[\pi^{*}\right]$ which encodes the number $w=4$ and the dynamic call-graph $G\left(P^{*}, I_{k e y}\right)$ of the watermarked program $P^{*}$.

Next, we first describe the data and operational components used by the model and, then, we present the embedding and the extracting watermarking algorithms.

\subsection{Model Components}

Our watermarking model uses two main categories of components namely data components and opera- 
tional components. The first category includes the dynamic call-graph $G\left(P, I_{k e y}\right)$ of the input $\operatorname{program} P$, the watermark graph $F\left[\pi^{*}\right]$, and the dynamic call-graph $G\left(P^{*}, I_{k e y}\right)$ of the watermarked program $P^{*}$, while the second category includes call patterns and control statements which are components related to the process of embedding the graph $F\left[\pi^{*}\right]$ into application program $P$.

We next describe the construction and main properties of the dynamic call-graph $G\left(P^{*}, I_{k e y}\right)$, two call patterns based on which we correspond edges of the call-graph $G\left(P^{*}, I_{k e y}\right)$ to function calls, and specific variables and statements which control the execution of real and water functions.

\section{(I) The Dynamic Call-graph $G\left(P^{*}, I_{k e y}\right)$}

Let $F\left[\pi^{*}\right]$ be a watermark-graph on $n+2$ nodes and $G\left(P, I_{k e y}\right)$ be the dynamic call-graph of a program $P$ on $n+3$ nodes $f_{\text {main }}, f_{s}, f_{1}, \ldots, f_{n}, f_{t}$ taken after running the program $P$ with the input $I_{k e y}$. In general, the selection of the input $I_{k e y}$ is such that it produces the call-graph $G\left(P, I_{k e y}\right)$ having structure as "close" as possible to the structure of $F\left[\pi^{*}\right]$. We assign the $n+2$ nodes $f_{s}=f_{n+1}, f_{n}, \ldots, f_{1}, f_{0}=f_{t}$ of the call-graph $G\left(P, I_{\text {key }}\right)$ to $n+2$ nodes $s=u_{n+1}, u_{n}, \ldots, u_{1}, u_{0}=t$ of $F\left[\pi^{*}\right]$ into 1-1 correspondence; the main function $f_{\text {main }}$ do not correspond to any node of $F\left[\pi^{*}\right]$.

Let $\left(u_{i}, u_{j}\right)$ be an edge in graph $F\left[\pi^{*}\right]$ and let $\left(f_{i}, f_{j}\right)$ be an edge in call-graph $G\left(P, I_{k e y}\right)$. We say that the edge $\left(f_{i}, f_{j}\right)$ corresponds to edge $\left(u_{i}, u_{j}\right)$ iff the node $f_{i}$ corresponds to $u_{i}$ and the node $f_{j}$ corresponds to $u_{j}, 0 \leq i, j \leq n+1$. Moreover, if $\left(u_{i}, u_{j}\right)$ is a forward (resp. backward) edge in the graph $F\left[\pi^{*}\right]$ we say that the corresponding edge $\left(f_{i}, f_{j}\right)$ in graph $G\left(P, I_{k e y}\right)$ is a forward (resp. backward) edge.

The dynamic call-graph $G\left(P^{*}, I_{k e y}\right)$ is constructed as follows:

- $V\left(G\left(P^{*}, I_{\text {key }}\right)\right)=V\left(G\left(P, I_{\text {key }}\right)\right)$, i.e., it has the same nodes as the call-graph $G\left(P, I_{k e y}\right)$;

- $E\left(G\left(P^{*}, I_{\text {key }}\right)\right)=E\left(F\left[\pi^{*}\right]\right)$, i.e., $\left(f_{i}, f_{j}\right)$ is an edge in $E\left(G\left(P^{*}, I_{k e y}\right)\right)$ iff the corresponding $\left(u_{i}, u_{j}\right)$ is an edge in $F\left[\pi^{*}\right]$.

The edges of the call-graph $G\left(P^{*}, I_{k e y}\right)$ are divided into two categories namely real and water edges; note that, the real (resp. water) edges are corresponded to real (resp. water) function calls. An edge $\left(f_{i}, f_{j}\right)$ of the call-graph $G\left(P, I_{k e y}\right)$ is characterized as either

- real edge if $\left(f_{i}, f_{j}\right)$ is an edge in $G\left(P, I_{k e y}\right)$, or

- water edge if $\left(f_{i}, f_{j}\right)$ is not an edge in $G\left(P, I_{k e y}\right)$.

Figure 2 shows the dynamic call-graph $G\left(P^{*}, I_{\text {key }}\right)$ along with its real edges (solid arrows) and water

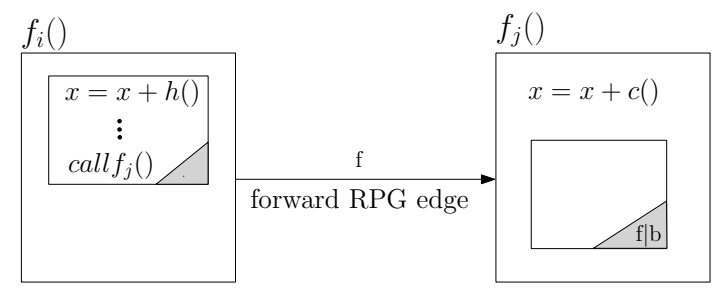

(a)

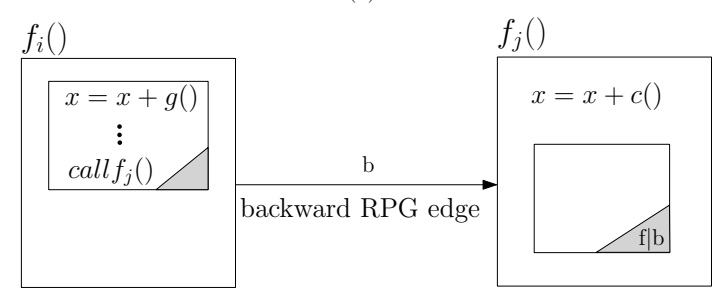

(b)

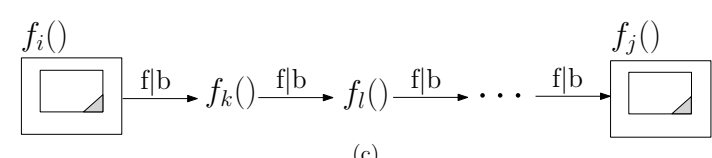

(c)

Figure 3: (a) The forward call pattern $f$-call; (b) The backward call pattern $b$-call; (c) The path call pattern $p$-call.

edges (dashed arrows); it also depicts the dynamic call-graph $G\left(P, I_{\text {key }}\right)$ and the watermark-graph $F\left[\pi^{*}\right]$.

\section{(II) Call Patterns}

In the implementation phase, we modify the source code of program $P$ using specific function call patterns which we describe below.

Let $P$ be an application program, $G\left(P, I_{\text {key }}\right)$ be the dynamic call-graph of the program $P$ with input $I_{k e y}$, and $F\left[\pi^{*}\right]$ be a watermark-graph which we have to embed into $P$. According to our watermarking model, the embedding process relies mainly on altering the execution-flow of appropriate function calls of $P$ such that the execution of the resulting program $P^{*}$ with the input $I_{k e y}$ produces a call-graph $G\left(P^{*}, I_{k e y}\right)$ which, after removing the node $f_{\text {main }}$, is isomorphic to watermark-graph $F\left[\pi^{*}\right]$.

Let $\left(f_{i}, f_{j}\right)$ be an edge of call-graph $G\left(P^{*}, I_{k e y}\right)$ or, equivalently, an edge which we want to appear in $G\left(P^{*}, I_{k e y}\right)$. Since $G\left(P^{*}, I_{k e y}\right)$ has two types of edges it follows that $\left(f_{i}, f_{j}\right)$ is either real or water edge. Based on the type of $\left(f_{i}, f_{j}\right)$, we do the following:

- if $\left(f_{i}, f_{j}\right)$ is a water edge we add the statement $\operatorname{call}\left(f_{j}\right)$ in the function $f_{i}$, while

- if $\left(f_{i}, f_{j}\right)$ is a real edge we add no call statement 
since the statement call $\left(f_{j}\right)$ exists in $f_{i}$.

Based on whether $\left(f_{i}, f_{j}\right)$ is either a forward or a backward edge we add specific statements in functions $f_{i}$ and $f_{j}$ according to the following two call patterns namely forward and backward call patterns:

(a) if $\left(f_{i}, f_{j}\right)$ is a forward edge we add the statement $x=x+h()$ in function $f_{i}$ before the call-site or, equivalently, call-point of the function $f_{j}$, and the statement $x=x+c()$ in the function $f_{j}$, while

(b) if $\left(f_{i}, f_{j}\right)$ is a backward edge we add the statement $x=x+g()$ in function $f_{i}$ before the call-site of the function $f_{j}$, and the statement $x=x+c()$ in the function $f_{j}$,

where $x$ is a variable of type $\mathrm{A}$ and $h(), g()$ and $c()$ functions which returns values of type A. Figure 3(a) depicts the forward call pattern or, for short, $f$-call, while Figure 3(b) depicts the backward call pattern or, for short, $b$-call.

Recall that the direct edge $\left(f_{i}, f_{j}\right)$ of a call-graph represents a function call operation where $f_{i}$ is the caller function and $f_{j}$ the callee function; in other words, it means that in function $f_{i}$ there exists the statement $\operatorname{call}\left(f_{j}\right)$. Hereafter, in this case we shall say that $\left(f_{i}, f_{j}\right)$ is a direct call.

In a call-graph of an application program we usually meet sequences of calls of the form $\left(f_{i}, f_{k_{1}}, f_{k_{2}}, \ldots, f_{k_{m}}, f_{j}\right)$. For simplicity we set $f_{i}=f_{k_{0}}$ and $f_{j}=f_{k_{m+1}}$ and suppose that each of these calls $\left(f_{k_{0}}, f_{k_{1}}\right),\left(f_{k_{1}}, f_{k_{2}}\right), \ldots,\left(f_{k_{m}}, f_{m+1}\right)$ is either forward or backward. We extend the notion of the direct call $\left(f_{i}, f_{j}\right)$ to indirect call $\left(f_{i} \rightarrow f_{j}\right)$; an indirect call consists of a path of functions $\left(f_{i}, f_{k_{1}}, \ldots, f_{j}\right)$ of length $\ell \geq 2$. Using the $\mathrm{f}$-call and b-call patterns, we next define the path call pattern or, for short, $p$-call as follows:

(c) if $\left(f_{k_{i}}, f_{k_{i+1}}\right)$ and $\left(f_{k_{i+1}}, f_{k_{i+2}}\right)$ are two consecutive calls of a call sequence, we apply an f-call or a b-call in $\left(f_{k_{i+1}}, f_{k_{i+2}}\right)$ by first adding the statement $x=x+h()$ or the statement $x=x+g()$ in the function $f_{k_{i+1}}$ after the call-point of statement $x=x+c()$, and then adding the statement $x=x+c()$ in the function $f_{k_{i+2}}, 0 \leq i \leq m-1$.

Figure 3 shows the structures of the patterns of an f-call and a b-call of the direct call $\left(f_{i}, f_{j}\right)$, and the structure of a p-call of an indirect call $\left(f_{i} \rightarrow f_{j}\right)$.

Note that an indirect call $\left(f_{i} \rightarrow f_{j}\right)$ consisting of a path of functions $\left(f_{i}, f_{k_{1}}, \ldots, f_{j}\right)$ of length $\ell$ can be considered as a sequence of $\ell$ direct calls.

\section{(III) Control Statements}

In any watermarking model both the original program $P$ and the watermarked program $P^{*}$ have to operate
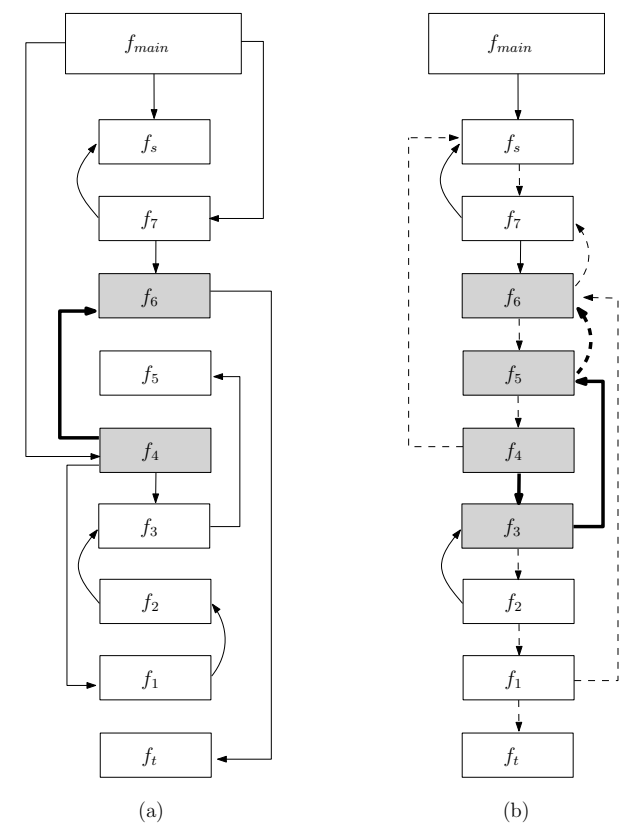

(b)

Figure 4: (a) The real-call $\left(f_{4}, f_{6}\right)$ in the call-graph $G\left(P, I_{k e y}\right)$ of a program $P$; bold arrow. (b) The corresponding path-call $\left(f_{4}, f_{3}, f_{5}, f_{6}\right)$ in the call-graph $G\left(P^{*}, I_{k e y}\right)$ of the watermarked program $P^{*}$; bold arrows.

identically, that is, the output $O(P, I)$ of the program $P$ must be the same with the output $O\left(P^{*}, I\right)$ of the program $P^{*}$ for every input $I$. Thus, since the callgraphs $G\left(P, I_{k e y}\right)$ and $G\left(P^{*}, I_{k e y}\right)$ dictate the execution flow of the original program $P$ and the watermarked program $P^{*}$, respectively, and since the call-graph $G\left(P, I_{\text {key }}\right)$ is not isomorphic to $G\left(P^{*}, I_{\text {key }}\right)$ we have to control the flow of selected function calls of $P^{*}$ so that $O(P, I)=O\left(P^{*}, I\right)$ for every input $I$.

To do this, we exploit the values of specific variables in a function $f_{i}$ by using them in some selected or added control statements as part of opaque predicates. More precisely, in our watermarking model we use the values of the variable $x$ of the f-call and b-call patterns and include it in a specific control statement $s$ causing thus an "appropriate execution flow" of the functions of the call-graph $G\left(P^{*}, I_{k e y}\right)$; with the term "appropriate execution flow" we mean that the execution flow of the functions of the call-graph $G\left(P^{*}, I_{k e y}\right)$ is such that $O(P, I)=O\left(P^{*}, I\right)$ for every input $I$.

Hereafter, we shall call $c f$-statement the control statement $s$ since it controls the execution flow of the functions of $G\left(P^{*}, I_{k e y}\right)$ and, in an analogous way, we shall call $c f$-variable the variable $x$ of the f-call and b-call patterns.

We next describe the mechanism which ensures 
an appropriate execution flow of the functions of $G\left(P^{*}, I_{k e y}\right)$ through the altering of the execution flow of the functions of the program $P$ by modifying or adding some specific control statements. In fact, what the mechanism actually does is to modify the conditions or expressions of these control statements by adding opaque predicates.

Definition 3.1. A predicate $Q$ is opaque at a program point $p$, if at point $p$ the outcome of $Q$ is known at embedding time. If $Q$ always evaluates to true we write $Q_{p}^{T}$, for false we write $Q_{p}^{F}$, and if $Q$ sometimes evaluates to true and sometimes to false we write $Q_{p}^{\text {? }}$.

Let $\left(f_{i}, f_{j}\right)$ be a direct call in our program $P^{*}$ or, equivalently, an edge in the call-graph $G\left(P^{*}, I_{\text {key }}\right)$; it is either real, water, forward, or backward edge. In any case, the proposed mechanism uses the value of the variable $x$ of the $\mathrm{f}$-call or b-call pattern of $\left(f_{i}, f_{j}\right)$ and does the following:

- In function $f_{i}$ : create a control statement (if, switch, for, while, etc), add an opaque predicate $Q_{p}^{?}$ with respect to cf-variable $x$ in the condition of the control statement, and insert it at a point $p$ before the statement $x=x+h()$ or $x=x+g()$; we can also select an existing control statement at a point $p$, consider it as cf-statement, and include in its condition part the opaque predicate $Q_{p}^{\text {? }}$.

- In function $f_{j}$ : create a control statement (if, switch, for, while, etc), add an opaque predicate $Q_{p}^{?}$ with respect to cf-variable $x$ in the condition of the control statement, and insert it at a point $p$ before the statement $x=x+c()$; the main body of $f_{j}$ is included in a block of a cf-statement the execution of which is depending upon the truthness or falsity of the opaque predicate $Q_{p}^{\text {? }}$. If $f_{j}$ is called by another function $f_{k}$, that is, if $\left(f_{k}, f_{j}\right)$ is an edge in the call-graph $G\left(P^{*}, I_{k e y}\right)$, we do not create a new control statement but we use the previous one and add or appropriately modify its existing opaque predicate $Q_{p}^{\text {? }}$.

Table 1 shows an example of the modification of the condition part of an if cf-statement via an opaque predicate; since $\left(f_{i}, f_{j}\right)$ is a water and forward function call, the statement $\operatorname{call}\left(f_{j}\right)$ does not exist in function $f_{i}$, and thus we add it in $f_{i}$, while the cfstatement is the $x=x+h()$. On the other hand, Table 2 shows an example in this case where $\left(f_{i}, f_{j}\right)$ is a real and backward function call. In this case, the statement $\operatorname{call}\left(f_{j}\right)$ does exist in $f_{i}$ while the cfstatement is the $x=x+g()$. Table 3 shows an example of the modification of the function $f_{j}$ in the case

\begin{tabular}{|c|c|}
\hline Program $P$ & Program $P^{*}$ \\
\hline \hline function $f_{i}()$ & function $f_{i}()$ \\
$\ldots$ & $\ldots$ \\
if (condition) & $\ldots$ \\
$\ldots$ & $\mathrm{x}=\mathrm{x}+\mathrm{h}() ;$ \\
statements; & $\ldots$ \\
$\ldots$ & call $f_{j}() ;$ \\
& $\ldots$ \\
& statements; \\
& $\ldots$ \\
\hline
\end{tabular}

Table 1: An example of cf-statement modification via opaque predicates in the case where $\left(f_{i}, f_{j}\right)$ is a water and forward function call.

\begin{tabular}{|c|c|}
\hline Program $P$ & Program $P^{*}$ \\
\hline function $f_{i}()$ & function $f_{i}()$ \\
\hline$\cdots$ & .. \\
\hline & if ( condition \& $Q_{p}^{?}$ ) \\
\hline if (condition) & $\ldots$ \\
\hline $\begin{array}{ll}\cdots \\
\text { call } f_{i}() \text {. }\end{array}$ & $x=x+g() ;$ \\
\hline $\begin{array}{l}\text { aded } \\
\ldots\end{array}$ & call $f_{j}()$; \\
\hline statements; & $\cdots$ \\
\hline$\cdots$ & statements; \\
\hline & $\ldots$ \\
\hline
\end{tabular}

Table 2: An example of cf-statement modification via opaque predicates in the case where $\left(f_{i}, f_{j}\right)$ is a real and backwards function call.

\begin{tabular}{|l|c|}
\hline Caller of $\left(f_{i}, f_{j}\right)$ & Callee of $\left(f_{i}, f_{j}\right)$ \\
\hline \hline function $f_{i}()$ & function $f_{j}()$ \\
$\ldots$ & $\ldots$ \\
if $\left(\right.$ condition \& $\left.Q_{p}^{?}\right)$ & if $\left(\right.$ condition $\left.\& Q_{p}^{?}\right)$ \\
$\ldots$ & $\ldots$ \\
$\mathrm{x}=\mathrm{x}+\mathrm{h}() ;$ & $\mathrm{x}=\mathrm{x}+\mathrm{c}() ;$ \\
$\ldots$ & $\ldots$ \\
call $f_{j}() ;$ & if $\left(\right.$ condition $\left.\& Q_{p}^{?}\right)$ \\
$\ldots$ & $\ldots$ \\
statements; & statements; \\
$\ldots$ & $\ldots$ \\
\hline
\end{tabular}

Table 3: An example of cf-statement modification via opaque predicates of the function $f_{j}$ in the case where $\left(f_{i}, f_{j}\right)$ is a water and forward function call.

where $\left(f_{i}, f_{j}\right)$ is a water and forward function call.

Remark 3.1. Based on the structural properties of the watermark graph $F\left[\pi^{*}\right]$ and call-graph $G\left(P^{*}, I_{k e y}\right)$ we 
can easily prove the following lemma.

Lemma 3.1. Let $G\left(P, I_{\text {key }}\right)$ and $G\left(P^{*}, I_{\text {key }}\right)$ be the callgraphs of programs $P$ and $P^{*}$, respectively, on input $I_{k e y}$, and let $\left(f_{i}, f_{j}\right)$ be an edge in call-graph $G\left(P, I_{k e y}\right)$. Then, there always exists an edge $\left(f_{i}, f_{j}\right)$ or a path $\left(f_{i}, f_{k_{1}}, f_{k_{2}}, \ldots, f_{k_{m}}, f_{j}\right)$ in call-graph $G\left(P^{*}, I_{k e y}\right)$.

Remark 3.2. In our implementation, in the case where $\left(f_{i}, f_{j}\right)$ is an edge in $G\left(P, I_{\text {key }}\right)$ and $\left(f_{i}, f_{j}\right)$ is not an edge in $G\left(P^{*}, I_{k e y}\right)$ we have to compute a path $\left(f_{i}, f_{k_{1}}, \ldots, f_{j}\right)$ of function calls in $G\left(P^{*}, I_{k e y}\right)$. Such a path is a shortest path from $f_{i}$ to $f_{j}$ in the graph $G\left(P^{*}, I_{\text {key }}\right)$; it may consist of all types of edges, that is, real or water edges and forward or backward edges. Figure 4(a) shows the edge $\left(f_{4}, f_{6}\right)$ in $G\left(P, I_{\text {key }}\right)$ which is not an edge in $G\left(P^{*}, I_{k e y}\right)$, while Figure 4(b) shows its corresponding shortest path from $f_{i}$ to $f_{j}$, that is, the path $\left(f_{4}, f_{3}, f_{5}, f_{6}\right)$; note that, $\left(f_{4}, f_{3}\right)$ is a real and forward edge, $\left(f_{3}, f_{5}\right)$ is a real and backward edge, and $\left(f_{5}, f_{6}\right)$ is a water and backward edge.

\section{(VI) Execution Rules}

We present the rules based on which we control the execution flow of the functions of $P^{*}$ such that $O(P, I)=O\left(P^{*}, I\right)$ for every input $I$. In fact, we show in all the cases how the value of $Q_{p}^{?}$ dictates the execution flow of functions of $G\left(P^{*}, I_{\text {key }}\right)$.

Let $\left(f_{i}, f_{j}\right)$ be a direct call in our program $P^{*}$ or, equivalently, an edge in the call-graph $G\left(P^{*}, I_{k e y}\right)$. We distinguish the following cases:

- Edge $\left(f_{i}, f_{j}\right)$ is real and forward/backward: in this case we modify the functions $f_{i}$ and $f_{j}$ as follows:

○ Function $f_{i}$ : the opaque predicate $Q_{p}^{\text {? }}$ in the cfstatement before the cf-value $x=x+h()$ or $x=$ $x+g()$ and the call $\left(f_{j}\right)$ is evaluated to true, that is, $Q_{p}^{T}$.

○ Function $f_{j}$ : the opaque predicate $Q_{p}^{\text {? }}$ in the cfstatement before the cf-value $x=x+c()$ is evaluated to true, that is, $Q_{p}^{T}$, while the $Q_{p}^{?}$ for the cf-statement which controls the statements of the main body of the function $f_{j}$ is also evaluated to true, that is, $Q_{p}^{T}$.

- Edge $\left(f_{i}, f_{j}\right)$ is water and forward/backward: in this case we modify the functions $f_{i}$ and $f_{j}$ as follows:

- Function $f_{i}$ : the opaque predicate $Q_{p}^{?}$ in the cfstatement before the cf-value $x=x+h()$ or $x=$ $x+g()$ and the call $\left(f_{j}\right)$ is evaluated to true, that is, $Q_{p}^{T}$.

○ Function $f_{j}$ : the opaque predicate $Q_{p}^{\text {? }}$ in the cfstatement before the cf-value $x=x+c()$ is eval- uated to true, that is, $Q_{p}^{T}$, while the $Q_{p}^{?}$ for the cf-statement which controls the statements of the main body of the function $f_{j}$ is evaluated to false, that is, $Q_{p}^{F}$.

Remark 3.3. At the execution time of the function $f_{i}$ of the program $P^{*}$, only one opaque predicate $Q_{p}^{?}$ in the cf-statements is evaluated to true with respect to the current value of the cf-variable $x$.

\subsection{Embedding an RPG into a Code}

Having described the main properties and operations of our dynamic watermarking model, let us now present the algorithm which efficiently watermark an application program $P$ by embedding the reducible permutation graph $F\left[\pi^{*}\right]$ into an application program $P$ producing thus the watermarked program $P^{*}$.

We next present in detail the proposed embedding algorithm, namely Encode_RPG.to.CODE, which consists of the following steps:

\section{Embedding Algorithm Encode_RPG.to.CODE}

1. Take as input the source code of the program $P$, select an input $I_{k e y}$, and construct the call-graph $G\left(P, I_{\text {key }}\right)$; let $f_{\text {main }}, f_{s}, f_{n}, \ldots, f_{1}, f_{t}$ be the functions of call-graph $G\left(P, I_{k e y}\right)$; then, construct a watermark graph $F\left[\pi^{*}\right]$ on $n+2$ nodes and let $s=u_{n+1}, u_{n}, \ldots, u_{1}, u_{0}=t$ be the nodes of $F\left[\pi^{*}\right]$;

2. Remove the node $f_{\text {main }}$ from $G\left(P, I_{k e y}\right)$ and assign an exact pairing, i.e., one-to-one and onto mapping, of the $n+2$ nodes of $G\left(P, I_{k e y}\right)$ to the nodes of $F\left[\pi^{*}\right]$; let $f_{i} \rightarrow u_{i}, 0 \leq i \leq n+1$;

3. Construct the call-graph $G\left(P^{*}, I_{k e y}\right)$ of the watermarked program $P^{*}$ as follows:

- $V\left(G\left(P^{*}, I_{k e y}\right)\right)=V\left(G\left(P, I_{k e y}\right)\right)$, i.e., it has the same nodes as the call-graph $G\left(P, I_{k e y}\right)$;

○ $E\left(G\left(P^{*}, I_{\text {key }}\right)\right)=E\left(F\left[\pi^{*}\right]\right)$, i.e., $\left(f_{i}, f_{j}\right)$ is an edge in $E\left(G\left(P^{*}, I_{k e y}\right)\right)$ iff the corresponding $\left(u_{i}, u_{j}\right)$ is an edge in graph $F\left[\pi^{*}\right]$;

4. Create a call-table $\mathcal{T}$ of dimension $(m \times 3)$, where $m$ is the number of function calls $\left(f_{i}, f_{j}\right)$ which appear in the execution trace of $P$ with input $I_{k e y}$; the 1 st column stores the caller functions $f_{i}$, the 2nd column stores the callee functions $f_{j}$, while the 3 rd one stores the invocations of $\left(f_{i}, f_{j}\right)$;

5. Initially insert into table $\mathcal{T}$ the edges $\left(f_{i}, f_{j}\right)$ of the call-graph $G\left(P^{*}, I_{k e y}\right)$; then, if $\left(f_{i}, f_{j}\right)$ does not exist in $G\left(P, I_{k e y}\right)$, compute the shortest path $\left(f_{i}, f_{k_{1}}, f_{k_{2}}, \ldots, f_{k_{m}}, f_{j}\right)$ from node $f_{i}$ to node $f_{j}$ 
in graph $G\left(P^{*}, I_{k e y}\right)$ and insert the edges $\left(f_{i}, f_{k_{1}}\right)$, $\left(f_{k_{1}}, f_{k_{2}}\right), \ldots,\left(f_{k_{m}}, f_{j}\right)$ into table $\mathcal{T}$; the edges are inserted into table $\mathcal{T}$ in a specific order;

6. Characterize the edges $\left(f_{i}, f_{j}\right)$ in table $\mathcal{T}$ as either real, water, forward, or backward;

7. For each water edge $\left(f_{i}, f_{j}\right)$ of the graph $G\left(P^{*}, I_{k e y}\right)$, add the statement $\operatorname{call}\left(f_{j}\right)$ in a callpoint in the function $f_{i}$ of the program $P$;

8. For each edge $\left(f_{i}, f_{j}\right)$ in the table $\mathcal{T}$, insert the cfvariable $x$ in both functions $f_{i}$ and $f_{j}$ by adding the statements $x=x+h(), x=x+g()$, or $x=x+c()$ according to f-call and b-call patterns; see, subsection (II) Call Patterns;

9. For each edge $\left(f_{i}, f_{j}\right)$ in the table $\mathcal{T}$, insert cfstatements which control:

$\circ$ the values of the cf-variables $x$,

$\circ$ the function calls call $\left(f_{j}\right)$, and

$\circ$ the values of the cf-variables $x$;

see, subsection (III) Control Statements;

10. Return the source code of the program $P^{*}$;

Remark 3.4. In Step 5 of the embedding algorithm, the edges $\left(f_{i}, f_{j}\right)$ are included into the table $\mathcal{T}$ in a specific order. This order is determined by the order they appeared in the execution trace of program $P$ with input $I_{k e y}$, that is, if call $\left(f_{i}, f_{j}\right)$ appears before the call $\left(f_{k}, f_{\ell}\right)$ in execution trace of $P$, the edge $\left(f_{i}, f_{j}\right)$ appears before the edge $\left(f_{k}, f_{\ell}\right)$ in table $\mathcal{T}$.

Remark 3.5. Let $\left(f_{i}, f_{j}\right)$ be an edge which is handled in Step 8 of the embedding algorithm and let the statement $\operatorname{call}\left(f_{j}\right)$ appears more that once in function $f_{i}$. We point out that in this case we insert both the cfvariable and cf-statement before the call-site of each statement $\operatorname{call}\left(f_{j}\right)$ in function $f_{i}$.

\subsection{Extracting the Watermark RPG from the Code}

In this section, we present an algorithm for extracting the graph $F\left[\pi^{*}\right]$ from the program $P^{*}$ watermarked by the embedding algorithm Encode_RPG.to.CODE. The proposed extracting algorithm works as follows:

Extracting Algorithm Decode_CODE.to.RPG

1. Take the program $P^{*}$ watermarked by the embedding algorithm Encode_RPG.to.CODE and run it with input $I_{k e y}$;
2. Construct the call-table $\mathcal{T}$ using the execution trace of the program $P^{*}$ with input $I_{k e y}$;

3. Construct the dynamic call-graph $G\left(P^{*}, I_{k e y}\right)$ using the call-table $\mathcal{T}$ as follows:

$\circ$ search the table $\mathcal{T}$ and add all the different functions $f_{i}$ in the set $V$, where $0 \leq i \leq n+2$,

- search the table $\mathcal{T}$ row-by-row and select all the different pairs $\left(f_{i}, f_{j}\right)$, where $f_{i}$ and $f_{j}$ belong to 1 st and 2 nd columns of the same row of $\mathcal{T}$, respectively; add the selected pairs $\left(f_{i}, f_{j}\right)$ in the set $E$;

$\circ$ take the vertex set $V\left(G\left(P^{*}, I_{k e y}\right)\right)=V$ and the edge set $E\left(G\left(P^{*}, I_{\text {key }}\right)\right)=E$ of the graph $G\left(P^{*}, I_{k e y}\right)$;

4. Remove the node $f_{\text {main }}$ from $G\left(P^{*}, I_{k e y}\right)$ resulting the graph $G^{\prime}\left(P^{*}, I_{k e y}\right)$;

5. Construct a graph $F\left[P^{*}\right]$ isomorphic to $G^{\prime}\left(P^{*}, I_{k e y}\right)$ and then compute the unique Hamiltonian path HP of the graph $F\left[P^{*}\right]$; let

$$
H P=\left(u_{n+1}, u_{n}, u_{n-1}, \ldots, u_{1}, u_{0}\right)
$$

be the Hamiltonian path of $F\left[P^{*}\right]$;

6. Relabeling the nodes of the graph $F\left[P^{*}\right]$ according to their order in the HP resulting thus the graph $F\left[\pi^{*}\right]$ with nodes $s=u_{n+1}, u_{n}, \ldots, u_{0}=t$;

7. Return the graph $F\left[\pi^{*}\right]$;

Remark 3.6. In Step 5 of the extracting algorithm, we compute the unique Hamiltonian path of the graph $F\left[P^{*}\right]$. Indeed, it has been shown that the reducible permutation graph $F\left[\pi^{*}\right]$ has always a unique Hamiltonian path, denoted by $\operatorname{HP}\left(F\left[\pi^{*}\right]\right)$, and this Hamiltonian path can be found in $O(n)$ time, where $n$ is the number of nodes of $F\left[\pi^{*}\right]$ (author's papers). Since $F\left[\pi^{*}\right]$ is isomorphic to $G^{\prime}\left(P^{*}, I_{k e y}\right)$ we can compute the unique Hamiltonian path HP of the graph $F\left[P^{*}\right]$ within the same time complexity.

\section{CONCLUDING REMARKS}

In this paper we presented a dynamic watermarking model for embedding a reducible permutation graph or, equivalently, a watermarking graph $F\left[\pi^{*}\right]$ into an application program $P$ using appropriate calls of specific functions of $P$. The main feature of our embedding model is its ability to embed the graph $F\left[\pi^{*}\right]$ into $P$ using only real functions and thus the size of the watermarked program $P^{*}$ remains relatively very 
small. Moreover, the proposed dynamic watermarking model has low time complexity and incorporates such properties which cause it resilient to attacks.

Our model uses opaque predicates in specific control statements in order to control the flow of selected function calls of $P^{*}$ so that the watermarked program $P^{*}$ have an appropriate execution, that is, $O(P, I)=$ $O\left(P^{*}, I\right)$ for every input $I$. It is worth noting that it is hard for an attacker to deduce an opaque predicate at run time. Specifically, the usage of opaque predicates in our model enables us to dictate the execution flow of function calls and also makes the programs' control flow difficult for an attacker to analyze it either statically or dynamically.

In addition, we point out that if an attacker makes a modification in some values of a cf-variable in a call-site $p$, he/she has to properly modify all the values of all the cf-variables in every call-site of the execution flow after $p$.

Moreover, an important property of our model is its ability to not use any mark during the embedding process in order to be able to extract the embedding watermark from the software.

Roughly speaking, the goal of an obfuscation technique is to make an attacker to hardly understand the operational parts of a code. Our model watermarks an application program $P$ in such a way that it withstands several obfuscation attacks among which layout and data obfuscation attacks. Moreover, the watermark can be effectively extracted even the code has been subjected to some control obfuscation attacks such as ordering (reordering expressions, reorder loops, etc). It is fair to mention, our model does not properly operate on some other control obfuscation attacks such as aggregation (inline functions, outline functions, etc).

Finally, in light of our dynamic watermarking model it would be very interesting to evaluate it in order to gain information on its practical behavior; we leave it as a problem for future work.

\section{REFERENCES}

Arboit, G. (2002). A method for watermarking java programs via opaque predicates. In Proc. 5th International Conference on Electronic Commerce Research (ICECR-5).

BSA (2011). 2011 BSA Global Software Piracy Study. Retrieved February, 2013, from http://portal.bsa.org/globalpiracy2011/.

Collberg, C., Carter, E., Debray, S., Huntwork, A., Kececioglu, J., Linn, C., and Stepp, M. (2004). Dynamic path-based software watermarking. In Proceedings of the ACM SIGPLAN 2004 conference on Programming language design and implementation, pages 107-118.

Collberg, C., Carter, E., Kobourov, S., and Thomborson, C. (2003). Error-correcting graphs for software watermarking. In Proc. 29th Workshop on Graphs in Computer Science ( $\left.W G^{\prime} 03\right)$, volume LNCS 2880, pages 156-167.

Collberg, C., Huntwork, A., Carter, E., Townsend, G., and Stepp, M. (2009). More on graph theoretic software watermarks: Implementation, analysis, and attacks. Information and Software Technology, 51:56-67.

Collberg, C. and Nagra, J. (2010). Surreptitious Software. Addison-Wesley.

Collberg, C. and Thomborson, C. (1999). Software watermarking: models and dynamic embeddings. In Proc 26th ACM SIGPLAN-SIGACT on Principles of Programming Languages (POPL'99), pages 311-324.

Collberg, C., Thomborson, C., Horning, J., Silbert, W., Matheson, L. R., Wright, A., and Owicki, S. (2011). Software watermarking techniques. US Patent, 2011/0214188

Cousot, P. and Cousot, R. (2004). An abstract interpretation-based framework for software watermarking. In Proc. 31st ACM SIGPLAN-SIGACT Symposium on Principles of Programming Languages (POPL'04), pages 173-185.

Cox, I., Kilian, J., Leighton, T., and Shamoon, T. (1996). A secure, robust watermark for multimedia. In Proc. 1st Int'l Workshop on Information Hiding, volume LNCS 1174 , pages 317-333.

Curran, D., Hurley, N., and Cinneide, M. (2003). Securing java through software watermarking. In Proc. Int'l Conference on Principles and Practice of Programming in Java (PPPJ'03), pages 145-148.

Davidson, R. and Myhrvold, N. (1996). Method and system for generating and auditing a signature for a computer program. US Patent, 5.559.884.

Graham, S., Kessler, P., and Mckusick, M. (1982). Gprof: A call graph execution profiler. SIGPLAN Not., 17(6):120-126.

Grover, D. (1997). The Protection of Computer Software Its Technology and Applications. Cambridge University Press, New York.

Hecht, M. and Ullman, J. (1972). Flow graph reducibilit. SIAM J. Computing, 1:188-202.

Hecht, M. and Ullman, J. (1974). Flow graph reducibilit. Journal of the ACM, 21:367-375.

Horne, W., Maheshwari, U., Tarjan, R., Horning, J., Silbert, W., Matheson, L. R., Wright, A., and Owicki, S. (2012). Systems and methods for watermarking software and other media. US Patent, 8.140.850.

Monden, A., Iida, H., Matsumoto, K., Inoue, K., and Torii, K. (2000). A practical method for watermarking java programs. In Proc. 24th Computer Software and Applications Conference (COMPSAC'00), pages 191197.

Moskowitz, S. and Cooperman, M. (1996). Method for stegacipher protection of computer code. US Patent, 5.745.569. 
Myles, G. and Collberg, C. (2006). Software watermarking via opaque predicates: implementation, analysis, and attacks. Electronic Commerce Research, 6:155-171.

Nagra, J. and Thomborson, C. (2004). Threading software watermarks. In Proc. 6th Int'l Workshop on Information Hiding (IH'04), volume LNCS 3200, pages 208223.

Qu, G. and Potkonjak, M. (1998). Analysis of watermarking techniques for graph coloring problem. In Proc. IEEE/ACM Int'l Conference on Computer-aided Design (ICCAD'98), volume ACM Press, pages 190193.

Rodriguez, T., MacIntosh, B., and Gustafson, A. (2010). Software watermarking. US Patent, 20100095376.

Sharma, B., Agarwal, R., and Singh, R. (2011). An efficient software watermark by equation reordering and fdos. In SocProS (2), volume 131, pages 735-745.

Stern, J., Hachez, G., Koeune, F., and Quisquater, J. (1999). Robust object watermarking: Application to code. In Proc. 3rd Int'l Workshop on Information Hiding (IH'99), volume LNCS 1768, pages 368-378.

Venkatesan, R., Vazirani, V., and Sinha, S. (2001). A graph theoretic approach to software watermarking. In Proc. 4th Int'l Workshop on Information Hiding (IH'O1), volume LNCS 2137, pages 157-168.

WIPO (2012). World Intellectual Property Indicators - 2012 Edition. Retrieved February, 2013, from http://www.wipo.int/ipstats/en/.

XiaoCheng, L. and Zhiming, C. (2010). Software watermarking algorithm based on register allocation. In Distributed Computing and Applications to Business Engineering and Science (DCABES), Ninth International Symposium on, pages 539-543.

Xie, T. and Notkin, D. (2002). An empirical study of java dynamic call graph extractors. In University of Washington CSE Technical Report 02-12-03.

Zhang, C., Wang, J., Thomborson, C., Wang, C., and Collberg, C. (2009). A semi-dynamic multiple watermarking schemefor java applications. In Proceedings of the nineth ACM workshop on Digital rights management, pages 59-72.

Zhang, L., Yang, Y., Niu, X., and Niu, S. (2003). A survey on software watermarking. Journal of Software, 14:268-277.

Zhang, X., Zhang, Z., and Zhang, C. (2011). Spread spectrum-based fragile software watermarking. In Nano, Information Technology and Reliability (NASNIT) 15th North-East Asia Symposium on, pages 150154.

Zhu, W., Thomborson, C., and Wang, F. (2005). A survey of software watermarking. In Proc. IEEE Int'l Conference on Intelligence and Security Informatics (ISI'05), volume LNCS 3495, pages 454-458. 